
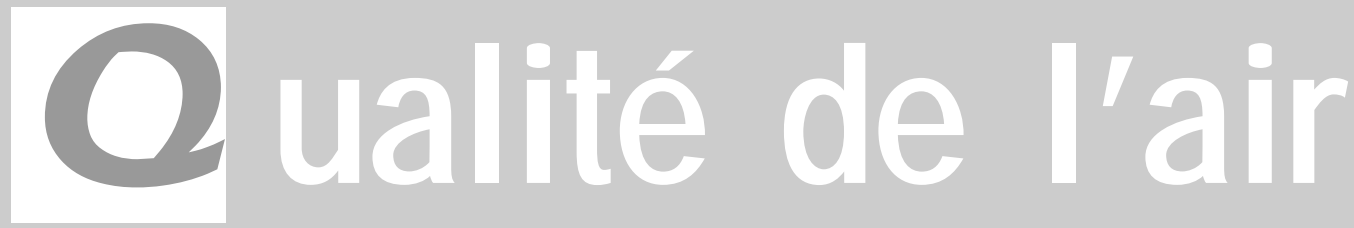

es problèmes de pollution ont aujourd'hui évolué en liaison avec la modification des activités humaines. L'opinion publique et l'ensemble des acteurs de la vie économique ont pris conscience de leur acuité. Au plan national, la récente loi sur l'air met l'accent sur le renforcement de la surveillance de la qualité de l'air et sur toute une série de dispositions juridiques et techniques visant l'amélioration à terme de la qualité de l'air dans les espaces de vie des populations.

Passage obligé dans bien des domaines, la mesure de la pollution de l'air s'effectue aujourd'hui non seulement au cœur des agglomérations urbaines où la pollution automobile constitue la préoccupation majeure, mais aussi à l'échelle périurbaine et même rurale, secteurs où la pollution d'origine urbaine se modifie par le biais de processus (photo)chimiques complexes.

Loin d'être une fin en soi, la mesure de la pollution de l'air doit répondre à des objectifs multiples : actions de surveillance à moyen

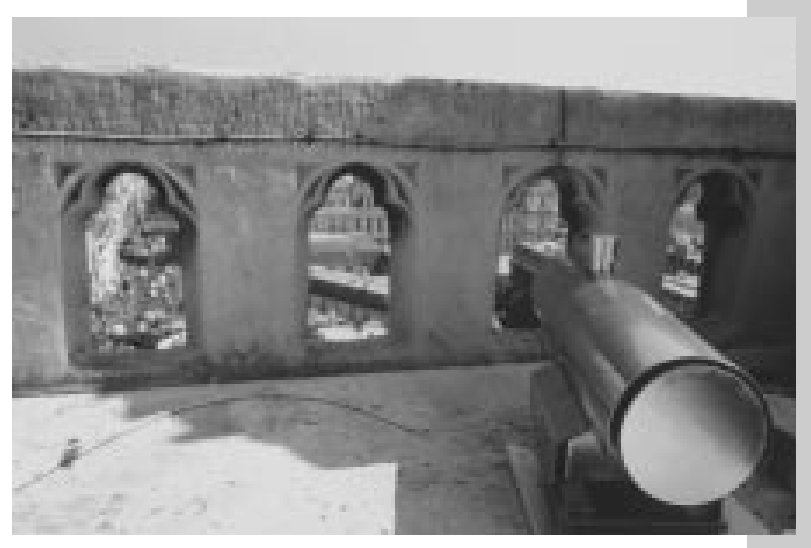

et long terme, actions de recherche pour une meilleure compréhension de la physicochimie atmosphérique, supports à la modélisation et aux enquêtes épidémiologiques, actions d'information et de prévision.

Les moyens à mettre en œuvre se diversifient au fil des années. Ils doivent permettre au plan spatio-temporel une caractérisation plus fine des environnements et de l'exposition des populations humaines qui y séjournent. Ils doivent fournir des renseignements plus pertinents par une palette élargie d'indicateurs en focalisant sur la mesure d'un nombre croissant de polluants toxiques à l'état de traces dans l'aérosol atmosphérique. Ils doivent aussi délivrer des réponses rapides au service des outils de prévision qui prendront de plus en plus d'importance au cours des années à venir.

Les différents articles de ce dossier consacrés à quelques grands thèmes de la pollution atmosphérique illustrent l'importance du rôle du laboratoire et de son instrumentation en aval des procédures de terrain nécessaires à la collecte des polluants gazeux ou particulaires dans l'environnement.

Nul doute que, dans un avenir proche, certaines techniques émergentes bénéficieront d'un succès grandissant. Citons par exemple, les dispositifs de télédétection optique, permettant de procéder, sans échantillonnage du milieu, à des mesures quasisimultanées et en temps réel en exploitant les propriétés optiques des gaz et particules. Dans un avenir plus lointain, parions aussi sur le développement de capteurs à couches sensibles intégrés à des dispositifs miniaturisés. L'objectif est qu'ils soient capables aussi bien dans l'environnement que dans l'évaluation de l'exposition individuelle, de délivrer des signaux qui puissent traduire, avec sensibilité et spécificité, les concentrations de toute une kyrielle d'aérocontaminants. 


\section{Qualité de l'air}

\section{9 Avant-Propos}

A. Person (LHNP, Paris)

M 11 L'aérosol atmosphérique : caractéristiques physico-chimiques

P. Masclet et H. Cachier (Laboratoire TEPE, ESIGEC, Université de Savoie, Le Bourget du Lac)

M 17 L'aérosol atmosphérique : analyse de la composante organique

$P$. Masclet et al. (Laboratoire TEPE, ESIGEC, Université de Savoie, Le Bourget du Lac)

M 21 Prélèvements des aérosols atmosphériques et analyses chimiques inorganiques J.P. Quisefit et al. (LISA, Universités Paris 7 et Paris 12, Créteil)

M 27 La mesure des particules en suspension dans l'air ambiant : applications dans les réseaux français de surveillance de la qualité de l'air F. Mathé et al. (École des Mines de Douai, Département Chimie et Environnement, Douai)

M 33 La métrologie des composés organiques volatils (COV) : méthodes et difficultés V. Jacob et al. (Groupe de Recherche sur l'Environnement et la Chimie Appliquée, Université Joseph Fourier, Grenoble)

M 38 Multidimensional capillary gas chromatography for the monitoring of individual non-methane hydrocarbons in air M. Veillerot et al. (Département Chimie et Environnement, École des Mines de Douai, Douai)

M 43 Les indicateurs de la pollution photochimique P.E Perros et T. Marion (Laboratoire Interuniversitaire des Systèmes Atmosphériques, Universités Paris 7 et 12, Créteil)

M 48 La mesure individuelle de l'exposition humaine aux polluants atmosphériques A. Person et al. (Laboratoire d'Hygiène de la Ville de Paris, Paris)

M 55 Évaluation technique des instruments à télédétection optique : DOAS et LIDAR T. Ménard et al. (INERIS, Parc Technologique Alata, Verneuil en Halatte) 